\title{
Avaliação da produção de bezerros em confinamento ou em suplementação exclusiva
}

\author{
[Evaluation of production of calves in feed lot or in creep feeding systems] \\ L.C.V. Ítavo ${ }^{1,7}$, C.C.B.F. Itavo ${ }^{2}$, S.R.M.B.O. Souza ${ }^{3}$, A.M. Dias ${ }^{4}$, E.M. Coelho ${ }^{1}$ \\ M.G. Morais ${ }^{5}$, F.F. Silva ${ }^{6,7}$ \\ ${ }^{1}$ Universidade Católica Dom Bosco \\ Av. Tamandaré, 6000 \\ 79117-900 - Campo Grande, MS \\ ${ }^{2}$ Aluna de pós-graduação - FMVZ-UNESP - Botucatu, SP \\ ${ }^{3}$ Aluna de graduação - UCDB - Campo Grande, MS \\ ${ }^{4}$ Universidade Estadual de Maringá - Maringá, PR \\ ${ }^{5}$ Universidade Federal de Mato Grosso do Sul - Campo Grande, MS \\ ${ }^{6}$ Universidade Estadual do Sudoeste da Bahia - Itapetinga, BA \\ ${ }^{7}$ Bolsista do CNPq
}

\begin{abstract}
RESUMO
Avaliou-se o desempenho de vacas e bezerros desmamados aos 90 (D90) e aos 210 (D210) dias, utilizando-se 36 vacas Beefalo-Nelore, multíparas e seus bezerros, em delineamento experimental de blocos ao acaso, com 18 repetições, sendo nove machos e nove fêmeas. Aos 90 dias pós-parto os bezerros foram pesados e distribuídos em dois tratamentos, adotando-se para a formação do bloco o peso e o sexo do bezerro. Os dois tratamentos foram creep-feeding (D210) e confinamento (D90). Os bezerros (D210) foram criados em pastagem de Brachiaria decumbens e $B$. humidicula com 48 hectares Os bezerros (D90) foram confinados em $7,95 \mathrm{~m}^{2} /$ animal e receberam capim elefante picado e concentrado. As médias de consumo de matéria seca do concentrado, do peso ao desmame e do ganho de peso foram 0,56 e 1,69kg/dia; 208,68 e 185,67kg; 0,75 e 0,52kg/dia, respectivamente, para bezerros D210 e D90. Matrizes D90 apresentaram maior peso $(448,86 \mathrm{~kg})$ aos 210 dias pós-parto. Sugere-se a suplementação excluviva, ou creep-feeding, para desmame de bezerros mais pesados.
\end{abstract}

Palavras-chave: bezerro de corte, creep-feeding, confinamento, desmame precoce, suplementação

\begin{abstract}
Performances of cows and calves weaned at 90 (D90) and 210 days of age (D210) were evaluated, using 36 Beefalo-Nelore multiparus cows and their calves, in a randomized block experimental design, with 18 replicates of nine animals of each sex. Calves were allotted to treatments using as criteria to form blocks, the weight and sex of the animal. Calves (D210) were raised in 48ha pasture of Brachiaria decumbens $e$ B. humidicula. Calves (D90) were raised in feedlot condition, in an area of $7.95 \mathrm{~m}^{2} /$ animal, and were fed chopped elephant grass and concentrate. The average concentrate dry matter intake, weaned body weight and weight gain were, respectively, 0.56 and $1.69 \mathrm{~kg} / \mathrm{day} ; 208.68$ and $185.67 \mathrm{~kg} ; 0.75$ and $0.52 \mathrm{~kg} /$ day respectively for D210 e D90 calves. Cows from D90 treatment showed higher body weight (448.86kg) at 210 days post partum. Creep-feeding is suggested for weaning heavier calves.
\end{abstract}

Keywords: beef calf, creep-feeding, feedlot, early weaning, supplementation

Recebido em 1 de novembro de 2005

Aceito em 14 de maio de 2007

* Autor para correspondência (corresponding author)

E-mail: itavo@ucdb.br 


\section{INTRODUÇÃO}

$\mathrm{O}$ trato digestivo de bezerros ao nascer assemelha-se fisiologicamente ao dos animais mamíferos não ruminantes. Nessa fase, o alimento básico é leite, sendo a atividade gástrica digestiva exercida pelo abomaso. É a fase mais crítica do ponto de vista nutricional, pois, devido às limitações enzimáticas e à ausência de síntese microbiana, o animal apresenta dificuldades em utilizar alimentos grosseiros, como volumosos. Segundo Rocha et al. (1999), o rúmen funcional é garantia de um animal capaz de aproveitar alimentos mais grosseiros. O tipo de alimentação ao qual o bezerro é submetido tem grande influência na transição do estado de préruminante para ruminante pleno. A técnica de alimentação suplementar denominada creepfeeding pode assumir grande importância e tornar-se indispensável para diminuir o tempo necessário ao acabamento dos animais. Segundo Sampaio et al. (2002), os benefícios do creepfeeding quanto à ingestão de nutrientes, consistem na adição de nutrientes à dieta do bezerro e não a substituição daqueles que naturalmente estariam presentes no leite e na forragem.

$\mathrm{Na}$ bovinocultura de corte, várias técnicas de desmame têm sido estudadas. O desmame precoce, realizado aos 60 a 90 dias após o parto, constitui uma prática que eleva a taxa de concepção. Dessa forma, o desmame precoce permite o direcionamento dos nutrientes da produção de leite para atender às exigências nutricionais da vaca. Vacas cujos bezerros foram desmamados precocemente apresentaram maior ganho de peso no período de serviço, maior taxa de prenhez e menor intervalo de partos em relação ao método tradicional de desmama (Restle et al., 1992). Uma outra forma de aumentar o peso à desmama, segundo Carvalho et al. (2003), seria fornecer suplemento em um comedouro privativo (creep-feeding).

O desempenho de animais cruzados depende do manejo nutricional, do ambiente e da distância genética entre as raças utilizadas. Por isso, faz-se necessário identificar aqueles cruzamentos que podem responder com maior produtividade para cada região do País. O Beefalo é um híbrido fértil, obtido pelo cruzamento do bisão americano com bovinos de origem européia, principalmente Charolês, Hereford e Angus, resultando em animais 3/8 bisão americano e 5/8 Bos taurus.

A produção do rebanho de cria é, sem dúvida, o componente mais importante da pecuária de ciclo completo. O desempenho reprodutivo de um rebanho está intimamente relacionado ao manejo, tanto sanitário quanto nutricional, os quais determinam as condições de peso e estado corporal da matriz e conseqüentemente o cio a cada início do período reprodutivo (Moojen et al., 1994). De acordo com o NRC (Nutrient..., 1996), a exigência energética de uma vaca cruzada lactante, dos 90 aos 180 dias pós-parto, é $57 \%$ maior do que de uma matriz não lactante. Quando a produção de leite cessa, a matriz inicia um processo de ganho de peso e melhora do estado corporal, que estimulam o retorno à reprodução (Restle e Vaz, 1998). A técnica de alimentação suplementar da cria pode proporcionar significativo descanso da matriz, resultando em melhoria da função reprodutiva, pois diminui a carga imposta pelo bezerro (Sampaio et al., 2002). Na busca de melhores índices de reprodução, técnicas de manejo também podem ser utilizadas, como o desmame antecipado de bezerros, causando interrupção na produção de leite e favorecendo a manifestação do cio (Restle et al., 2001).

Os objetivos deste trabalho foram avaliar o desempenho e o consumo de nutrientes em bezerros em confinamento, após o desmame aos 90 dias e em pastagens suplementados (creepfeeding) até 210 dias de idade, e estudar a variação do peso corporal das matrizes, entre 90 e 210 dias após o parto.

\section{MATERIAL E MÉTODOS}

O experimento foi realizado entre os meses de abril e agosto de 2003, utilizando-se 36 animais, dos quais 18 eram machos e 18 eram fêmeas, com 90 dias de média de idade, distribuídos em dois tratamentos: em pastagem (creep-feeding, D210) e em confinamento (desmame precoce, D90). No D210 utilizou-se uma área de 40 hectares formada por Brachiaria decumbens e Brachiaria humidicula, subdividida em três piquetes e equipada com módulo de alimentação seletiva, para viabilizar o arraçoamento seletivo dos bezerros. No D90, usou-se uma área de $254,7 \mathrm{~m}^{2}$, dividida em duas, a fim de acomodar 
machos e fêmeas, separadamente, com alternância a cada pesagem, até completarem 210 dias de idade. Nesse grupo o desmame ocorreu aos 90 dias. Os animais foram pesados a cada 28 dias até a idade de 210 dias.

O concentrado utilizado nos dois tratamentos era composto por milho em grão moído, farelo de soja, sorgo e núcleo mineral vitamínico e foi fornecido à vontade. As sobras de alimento do comedouro foram quantificadas uma vez por semana, a fim de estimar a ingestão do concentrado pelos animais no sistema creep- feeding e, diariamente, no sistema de confinamento. Foram coletadas amostras e analisadas periodicamente quanto aos teores de matéria seca (MS), proteína bruta $(\mathrm{PB})$, extrato etéreo (EE), matéria orgânica (MO), matéria mineral (MM), fibra em detergente neutro (FDN) e fibra em detergente ácido (FDA) segundo metodologias descritas por Silva e Queiroz (2002). A composição químico-bromatológica do concentrado utilizado no creep-feeding e no confinamento e do capim-elefante (Pennisetum purpureum Schum. cv. Napier) utilizado no confinamento são apresentados na Tab. 1.

Tabela 1. Composição químico-bromatológica do concentrado utilizado no creep-feeding e no confinamento e do capim-elefante (Pennisetum purpureum Schum. cv. Napier) utilizado no confinamento

\begin{tabular}{lcccccc}
\hline \multirow{2}{*}{ Alimento } & MS & MO & PB & EE & FDN & FDA \\
\cline { 3 - 7 } & & $(\%)$ & & & $\%$ da MS & \\
\hline Capim-elefante & 22,11 & 93,70 & 5,62 & 1,24 & 79,22 & 50,07 \\
Concentrado & 92,26 & 91,61 & 17,83 & 1,90 & 43,75 & 11,93 \\
\hline
\end{tabular}

MS: matéria seca; MO: matéria orgânica, PB: proteína bruta ( $\mathrm{N}$ total x 6,25); EE: extrato etéreo; FDN: fibra em detergente neutro; FDA: fibra em detergente ácido.

Foi utilizado um delineamento experimental em blocos ao acaso, os resultados em função do peso e sexo do bezerro, para estudar as variáveis consumo de nutrientes, peso dos bezerros e ganho médio diário em função do sexo dentro dos sistemas, e para comparar o consumo de concentrado nos sistemas.

Para avaliar o desempenho de matrizes foram utilizadas 36 vacas Beefalo-Nelore, multíparas, pesadas aos 90 dias pós-parto e distribuídas ao acaso entre os tratamentos, em função do peso e sexo de seus bezerros. Dezoito vacas foram submetidas ao sistema de desmame precoce $(90$ dias pós-parto), sendo seus bezerros encaminhados para o sistema D90, e 18 para o sistema de creep-feeding. Estas permaneceram com seus bezerros.

Todas as matrizes permaneceram em uma área de pastejo de 48 hectares formada por Brachiaria decumbens e Brachiaria humidicula, subdividida em três piquetes. Os piquetes eram providos de comedouros e bebedouros, sendo que a água e a suplementação mineral foram fornecidas à vontade. A área de pastejo era equipada com um módulo de alimentação seletiva, em ferro galvanizado, instalado na área de descanso dos animais, para viabilizar o arraçoamento seletivo dos bezerros (creep-feeding), de tal modo que as matrizes ficassem impedidas de alcançar o cocho de alimentação exclusiva. $\mathrm{O}$ experimento teve a duração de 120 dias.

As vacas foram pesadas ao início do experimento e a cada 28 dias, até 210 dias pós-parto. Para comparação entre a variação de peso das matrizes foi utilizado o teste F no nível de 5\% de significância. Para as análises estatísticas utilizou-se o SAEG (Sistema..., 1997).

\section{RESULTADOS E DISCUSSÃO}

Na Tab. 2 apresenta-se o consumo de nutrientes do concentrado em função do sistema de produção. Houve diferença entre os tratamentos: os bezerros do D210 consumiram menos MS e $\mathrm{PB}$ em $\mathrm{kg} / \mathrm{dia}$ do que os do D90. Isso pode ser atribuido ao fato de os animais do sistema creepfeeding terem recebido leite materno para suprir suas exigências nutricionais, além do concentrado. Aos 210 dias de idade, os bezerros do D210 pesaram $208,68 \mathrm{~kg}$, em média, enquanto que os do D90, $185,67 \mathrm{~kg}$. A diferença foi de $23,01 \mathrm{~kg}$.

No confinamento os machos apresentaram, aos 210 dias, ganho médio diário (GMD) de $0,60 \mathrm{~kg} / \mathrm{dia}$, e as fêmeas, $0,53 \mathrm{~kg} / \mathrm{dia}$, mas a diferença não foi significativa $(\mathrm{P}>0,05)$. O mesmo aconteceu com os animais do creep- 
feeding, cujos ganhos foram 0,78 e 0,71g/dia, respectivamente, para machos e fêmeas.

$\mathrm{Na}$ Tab. 3 são apresentados os resultados de consumo de nutrientes do concentrado em função do sexo para o sistema de confinamento. Não houve diferença entre machos e fêmeas $(\mathrm{P}>0,05)$. Estes resultados assemelham-se aos obtidos por Sampaio et al. (2002), ao utilizarem 5 e $10 \%$ de $\mathrm{NaCl}$ no suplemento, como alternativa para viabilizar o sistema de alimentação de bezerros em creep-feeding. Os autores observaram consumo de suplemento de 0,72 e $0,47 \mathrm{~kg} /$ animal $/$ dia e médias de peso à desmama iguais a 208,40 e $223,00 \mathrm{~kg}$, respectivamente, para os níveis 5 e $10 \%$ de inclusão de $\mathrm{NaCl}$, e concluíram que a adição de $10 \%$ de $\mathrm{NaCl}$ ao concentrado do creep-feeding proporcionou aumento de ganho de peso corporal em relação à adição de $5 \%$.

Tabela 2. Médias de consumos de matéria seca (CMS), proteína bruta (CPB), fibra em detergente neutro $(\mathrm{CFDN})$, fibra em detergente ácido (CFDA) do concentrado, em quilos por dia ( $\mathrm{kg} / \mathrm{dia})$, em porcentagem de peso vivo (\%PV) e em gramas por dia de peso metabólico $\left(\mathrm{g} / \mathrm{kg} \mathrm{PV}^{0,75}\right)$, de bezerros dos 90 (D90) aos 210 (D210) dias de idade, de acordo com os tratamentos

\begin{tabular}{|c|c|c|}
\hline Variável & $\begin{array}{l}\text { Creep-feeding } \\
\text { (D210) }\end{array}$ & $\begin{array}{l}\text { Confinamento } \\
\text { (D90) }\end{array}$ \\
\hline CMS (kg/dia) & $0,56 \mathrm{~b}$ & $1,69 \mathrm{a}$ \\
\hline CPB (kg/dia) & $0,10 \mathrm{~b}$ & $0,30 \mathrm{a}$ \\
\hline CFDN (kg/dia) & $0,24 b$ & $0,74 a$ \\
\hline CFDA (kg/dia) & $0,07 \mathrm{~b}$ & $0,20 \mathrm{a}$ \\
\hline $\mathrm{CEE}(\mathrm{kg} / \mathrm{dia})$ & $0,01 \mathrm{~b}$ & $0,03 \mathrm{a}$ \\
\hline CMS (\%PV) & $0,29 b$ & $1,04 \mathrm{a}$ \\
\hline CFDN (\%PV) & $0,13 b$ & $0,46 a$ \\
\hline CFDA (\%PV) & $0,03 b$ & $0,12 \mathrm{a}$ \\
\hline CMS (g/kg PV & $10,82 b$ & $37,09 a$ \\
\hline $\mathrm{CFDN}\left(\mathrm{g} / \mathrm{kg} \mathrm{PV}^{0,75}\right)$ & $4,73 b$ & $16,23 \mathrm{a}$ \\
\hline CFDA $\left(\mathrm{g} / \mathrm{kg} \mathrm{PV}^{0,75}\right)$ & $1,30 \mathrm{~b}$ & $4,44 a$ \\
\hline
\end{tabular}

Médias seguidas por letras distintas na mesma linha diferem entre si pelo teste $\mathrm{F}(\mathrm{P}<0,05)$.

O consumo de nutrientes do concentrado no Sistema creep-feeding foi avaliado para machos e fêmeas juntos, assim, não houve como determinar diferenças entre sexos quanto ao consumo de matéria seca e proteína bruta, que foram de 0,56 e $0,10 \mathrm{~kg} / \mathrm{dia}$, respectivamente. $\mathrm{O}$ consumo de MS em porcentagem de peso vivo foi de 0,29 para machos e fêmeas. Provavelmente, isso se deve ao fato de os machos ainda não terem entrado na puberdade e, por isso, foram considerados iguais até essa idade.

Oliveira (2003) observou melhor desempenho de bezerros criados até a desmama em pastagens mistas $(\mathrm{P}<0,05)$ quando comparados aos criados em pastagens nativas e/ou cultivadas de Brachiaria decumbens e $B$. humidicola. Provavelmente, segundo o autor, esse melhor desempenho pode ser atribuido ao fato de a pastagem mista oferecer maior oportunidade de seleção do pasto tanto pelas matrizes como pelos bezerros. Assim, as vacas poderiam selecionar melhor sua dieta a fim de suprir suas exigências nutricionais para mantença e produção de leite.

Tabela 3. Médias de consumos de matéria seca (CMS), proteína bruta (CPB), fibra em detergente neutro (CFDN), fibra em detergente ácido (CFDA) e extrato etéreo do concentrado, em $\mathrm{kg} / \mathrm{dia}$, em \% peso vivo (PV) e em g/dia de peso metabólico $\left(\mathrm{g} / \mathrm{kg} \mathrm{PV}^{0,75}\right)$ de bezerros dos 90 aos 210 dias de idade, em confinamento, de acordo com o sexo

\begin{tabular}{|c|c|c|}
\hline Variável & Macho & Fêmea \\
\hline CMS (kg/dia) & 1,77 & 1,60 \\
\hline CPB (kg/dia) & 0,32 & 0,29 \\
\hline CFDN (kg/dia) & 0,77 & 0,70 \\
\hline CFDA (kg/dia) & 0,21 & 0,20 \\
\hline $\mathrm{CEE}(\mathrm{kg} / \mathrm{dia})$ & 0,03 & 0,03 \\
\hline CMS (\%PV) & 1,07 & 1,01 \\
\hline CFDN (\%PV) & 0,47 & 0,44 \\
\hline CFDA (\%PV) & 0,13 & 0,12 \\
\hline CMS (g/kg PV $\left.{ }^{0,75}\right)$ & 38,34 & 35,84 \\
\hline CFDN $\left(\mathrm{g} / \mathrm{kg} \mathrm{PV}{ }^{0,75}\right)$ & 16,77 & 15,68 \\
\hline CFDA $\left(\mathrm{g} / \mathrm{kg} \mathrm{PV}^{0,75}\right)$ & 4,59 & 4,29 \\
\hline
\end{tabular}


Na Tab. 4 são apresentados os pesos iniciais aos 90 dias e finais aos 210 dias dos bezerros em função do sistema de produção. Os animais desmamados aos 90 dias apresentaram menor ganho de peso $(\mathrm{P}<0,05)$ que os que permaneceram na pastagem com suas mães.

Tabela 4. Peso vivo aos 90 (PV90) e aos 210 dias de idade (PV210) e ganho médio diário (GMD) de bezerros Beefalo-Nelore, em função do sistema de produção

\begin{tabular}{lcc}
\hline Variável & $\begin{array}{c}\text { Creep-feeding } \\
\text { (D210) }\end{array}$ & $\begin{array}{c}\text { Confinamento } \\
\text { (D90) }\end{array}$ \\
\hline PV90 $(\mathrm{kg})$ & $118,66 \mathrm{a}$ & $122,89 \mathrm{a}$ \\
PV210 $(\mathrm{kg})$ & $208,68 \mathrm{a}$ & $185,67 \mathrm{~b}$ \\
GMD $(\mathrm{kg} /$ dia) & $0,75 \mathrm{a}$ & $0,52 \mathrm{~b}$
\end{tabular}

Médias seguidas por letras distintas na mesma linha diferem entre si pelo teste $\mathrm{F}(\mathrm{P}<0,05)$.

O fato de os bezerros do D90 apresentarem menor desempenho pode ser atribuído à capacidade de aproveitamento de volumoso, pois esses animais ainda seriam altamente dependentes do leite para suprir suas exigências nutricionais. De acordo com Restle e Vaz (1998), o desmame precoce pode prejudicar o adequado desenvolvimento do bezerro. Segundo Rocha et al. (1999), o rúmen funcional garante o aproveitamento de alimentos mais grosseiros, embora com menor eficiência. Todavia, o rúmen tem pequena importância na nutrição do bezerro que recebe o leite como seu único alimento. Tais aspectos justificariam o menor desempenho dos bezerros mantidos em confinamento, desmamados precocemente, comparados aos que tiveram acesso ao leite materno até os 210 dias. Alencar et al. (1998), ao trabalharem com avaliação de características de crescimento até a desmama de bovinos da raça Nelore e cruzados Nelore-Charolês, em regime exclusivo de pasto e recebendo suplementação mineral, verificaram, aos 210 dias, peso vivo médio de 184,13 e $212,74 \mathrm{~kg}$ para animais puros e cruzados $(5 / 8$ Charolês $\mathrm{x} 3 / 8$ Nelore), respectivamente, e verificaram que os cruzados tiveram peso maior à desmama que os animais puros. Os valores da Tab. 4 assemelham-se aos citados por esses autores.

$\mathrm{Na}$ Tab. 5 são apresentados os resultados de desempenho dos bezerros dos 90 aos 210 dias de idade em função do sexo. Não se observou diferença no PV e no GMD de peso entre machos e fêmeas aos 90 e aos 210 dias de idade, nos dois tratamentos.

Tabela 5. Peso vivo aos 90 (PV90) e peso vivo aos 210 dias de idade (PV210) e ganho médio diário (GMD) de bezerros Beefalo-Nelore por sistema de produção, em função do sexo

\begin{tabular}{lrr}
\hline & Macho & Fêmea \\
\hline Confinamento & & \\
PV90 (kg) & 123,89 & 121,89 \\
PV210 (kg) & 196,11 & 185,67 \\
GMD (kg/dia) & 0,60 & 0,53 \\
Creep-feeding & & \\
PV90 (kg) & 116,15 & 121,44 \\
PV210 (kg) & 210,30 & 206,89 \\
GMD (kg/dia) & 0,78 & 0,71 \\
\hline
\end{tabular}

Perotto et al. (2001), ao estudarem o peso à desmama e o ganho de peso do nascimento à desmama de bovinos Charolês, Caracu e seus cruzamentos, verificaram desmama de bezerros com 208 dias de idade e com média de peso de $165 \mathrm{~kg}$. Tal valor é menor que o obtido aos 210 dias, para ambos os sistemas de produção avaliados. Esses autores, entretanto, não suplementaram os bezerros. Na prática, a decisão de suplementar ou não dependerá fundamentalmente do estado nutricional e do crescimento observado nos bezerros, assim como da condição corporal das matrizes, do custo de produção, da facilidade de manejo e do desempenho esperado dos animais

$\mathrm{Na}$ Tab. 6 são apresentados os resultados de desempenho produtivo das matrizes, em função do sistema de produção de seus bezerros. O peso vivo aos 210 dias pós-parto diferiu $(\mathrm{P}<0,05)$ em função dos sistemas de produção adotados. As vacas que permaneceram com bezerros por 210 dias no sistema D210 tiveram peso menor que as do sistema D90, aos 90 dias. Tal situação pode estar relacionada ao fato de as matrizes permanecerem com seus bezerros por um período mais longo e, por isso, tiveram que mobilizar nutrientes que poderiam ser utilizados para o aumento de peso e para a produção de leite. Estes resultados assemelham-se aos de Restle et al. (2001), que verificaram maior peso das matrizes Charolês-Nelore cujos bezerros foram desmamados aos 90 dias, em comparação com aquelas que permaneceram com bezerros por 210 dias (384 vs $339 \mathrm{~kg})$. 
Tabela 6. Peso vivo aos 90 dias (PV90) e aos 210 dias pós-parto (PV210) e ganho médio diário (GMD) de matrizes, em função do sistema de produção de seus bezerros

\begin{tabular}{lcc}
\multicolumn{1}{c}{ Variável } & $\begin{array}{c}\text { Creep-feeding } \\
\text { (D210) }\end{array}$ & $\begin{array}{c}\text { Desmame } \\
\text { precoce (D90) }\end{array}$ \\
\hline PV90 (kg) & $433,50 \mathrm{a}$ & $428,25 \mathrm{a}$ \\
PV210 (kg) & $424,71 \mathrm{a}$ & $448,86 \mathrm{~b}$ \\
GMD (kg/dia) & $-0,07 \mathrm{a}$ & $0,17 \mathrm{~b}$ \\
\hline
\end{tabular}

Médias seguidas por letras distintas na mesma linha diferem entre si pelo teste $\mathrm{F}(\mathrm{P}<0,05)$

Almeida et al. (2002), ao estudarem época de desmame e desempenho reprodutivo de vacas de corte constataram que as submetidas ao desmame precoce tiveram mais peso $(11,1 \mathrm{~kg})$ que as submetidas ao desmame tradicional, provavelmente devido à redução da exigência nutricional, anteriormente destinada à lactação, o que possibilitou maiores ganhos de peso.

O GMD diferiu $(\mathrm{P}<0,05)$ em função do sistema de produção. As vacas do D210 perderam, em média, $0,07 \mathrm{~kg} / \mathrm{dia}$, e as do D90 ganharam em média, $0,17 \mathrm{~kg} / \mathrm{dia}$. Estes resultados assemelhamse aos de Restle et al. (2001), que observaram maior ganho de peso diário das matrizes cujos bezerros foram desmamados aos 90 dias $(0,178$ vs $0,183 \mathrm{~kg})$. Esses autores atribuíram esses resultados à deficiência nutricional das pastagens, que não foi capaz de suprir às exigências de mantença, produção de leite e ganho de peso durante o período de aleitamento. Isso pode ter acontecido neste experimento.

A diferença entre o ganho de peso de vacas lactantes e não lactantes é o resultado do deficit na exigência energética. Segundo o NRC (Nutrient..., 1996), este ciclo de perda e ganho de peso, embora biologicamente eficiente, pode ser mais econômico e não causar danos à produção total, dependendo da duração e da severidade das perdas.

Ítavo et al. (2005) avaliaram o efeito do tamanho da vaca sobre o peso ao desmame de seus bezerros e observaram efeito da idade da vaca sobre o peso ao desmame, o que proporcionou aumento linear de $0,80 \mathrm{~kg}$ no peso de bezerros, com o aumento da idade da vaca ao parto. Tal fato sugere que as vacas mais velhas seriam indicadas para a produção de bezerros mais pesados. Todavia, os autores verificaram efeito linear negativo para a relação de desmame $(\mathrm{kg}$ de bezerro desmamado/kg de vaca ao desmame), que representou $1,2 \%$ de redução por ano de idade da vaca. Segundo os autores, esses resultados sugerem que a utilização de vacas jovens é mais eficiente para a produção de bezerros mais pesados. Essa informação associada ao uso de animais de elevado potencial de ganho de peso e ao uso de suplementação durante a fase de crescimento, corresponderia ao sucesso do sistema de produção de bovinos jovens para abate.

Segundo o NRC (Nutrient..., 1996), a exigência de energia de mantença da vaca em lactação é maior que a da não lactante. Ao cessar a lactação, o organismo animal utiliza os nutrientes para o ganho de peso e, conseqüentemente, melhora o estado corporal (Restle et al., 2001), fato observado nas vacas do sistema creepfeeding (Tab. 6). Nesse sistema ocorreu menor consumo de nutrientes proveniente do concentrado. Não houve efeito do sexo para consumo de nutrientes no sistema de confinamento. Ainda, os animais do creepfeeding tiveram maiores pesos aos 210 dias e houve maior ganho de peso diário que os confinados, desmamados precocemente.

Não houve efeito do sexo no desenvolvimento ponderal dos bezerros nos sistemas de produção avaliados. As matrizes cujos bezerros foram desmamados precocemente apresentaram maior peso vivo médio no final, aos 210 dias pós-parto.

\section{CONCLUSÕES}

Recomenda-se o uso da suplementação exclusiva de bezerros para incremento do peso corporal ao desmame e da relação peso do bezerro:peso da vaca. A antecipação do desmame para 90 dias de idade favorece a recuperação do peso e da condição corporal da vaca.

\section{REFERÊNCIAS BIBLIOGRÁFICAS}

ALENCAR, M.M.; TREMATORE, R.L.; OLIVEIRA, J.A.L. et al. Características de crescimento até a desmama de bovinos da raça Nelore e cruzados Charolês-Nelore. Rev. Bras. Zootec., v.27, p.40-46, 1998.

ALMEIDA, L.S.P.; LOBATO, J.F.P.; SCHENKEL, F.S. Data de desmame e desempenho reprodutivo de vacas de corte. Rev. Bras. Zootec., v.31, p.1223-1229, 2002. 
CARVALHO, F.A.N.; BARBOSA, F.A.; McDOWELL, L.R. (Eds). Nutrição de bovinos a pasto, Belo Horizonte: Papelform Editora, 2003. 438p.

ÍTAVO, L.C.V.; EUCLIDES FILHO, K. TORRES JR., R.A.A. et al. Eficiência de produção de bezerros de vacas de diferentes tamanhos. In: CONGRESSO INTERNACIONAL DE ZOOTECNIA, 7., 2005, Campo Grande. Anais... Campo Grande: UEMS:ZOOTEC, 2005.

MOOJEN, J.G.; RESTLE, J.; MOOJEN, E.L. et al. Efeito da época da desmama e da pastagem no desempenho de vacas e terneiros de corte. Desempenho de terneiros. Ciên. Rural, v.24, p.399403, 1994.

NUTRIENT requeriments of beef cattle. Washington, DC: National Academy of Sciences, 1996. 242p.

OLIVEIRA, N.P.R. Avaliação do vitelo orgânico do pantanal como alternativa de desenvolvimento local sustentável. 2003. 52f. Dissertação (Mestrado) Universidade Católica Dom Bosco, Campo Grande, MS.

PEROTTO, D.; ABRAHÃO, J.J.S.; KROETZ, I.A. Produtividade à desmama de novilhas Nelore e F1 Bos taurus x Nelore e Bos indicus x Nelore. Rev. Bras. Zootec., v.30, p.1712-1719, 2001

RESTLE, J.; DALLA PORTA, M.A.; SENNA, D.B. et al. Desempenho reprodutivo de novilhas de diferentes grupos genéticos submetidas previamente a duas idades de desmame. In: REUNIÃO ANUAL DA SOCIEDADE BRASILEIRA DE ZOOTECNIA, 29., 1992, Lavras. Anais... Lavras: SBZ, 1992. p.219

RESTLE, J.; VAZ. R.Z. Desmame precoce de bezerros. In: SIMPÓSIO INTERNACIONAL SOBRE PRODUÇÃO INTENSIVA DE GADO DE CORTE. 2., 1998, São Paulo. Anais... São Paulo. 1998. p.3-9.

RESTLE, J.; VAZ. R.Z.; ALVES FILHO, D.C.A. et al. Desempenho de vacas Charolês e Nelore desterneiradas aos três ou sete meses. Rev. Bras. Zootec., v.30, p.499-507, 2001.

ROCHA, E.O.; FONTES, C.A.A.; PAULINO, M.F. et al. Influência da idade de desmama e de início do fornecimento do volumoso a bezerros sobre a digestibilidade de nutrientes e o balanço de nitrogênio, pós-desmama. Rev. Bras. Zootec., v.28, p.143-147, 1999.

SAMPAIO, A.A.M.; BRITO, R.M.; CRUZ, G.M. et al. Utilização de $\mathrm{NaCl}$ no suplemento como alternativa para viabilizar o sistema de alimentação de bezerros em creep-feeding. Rev. Bras. Zootec., v.31, p.164-172, 2002.

SILVA, D.J.; QUEIROZ, A.C. Análises de alimentos, métodos químicos e biológicos. 3.ed. Viçosa: UFV, 2002. 235p.

SISTEMA de análises estatísticas e genéticas - SAEG. Viçosa: UFV, 1997. 